\title{
Evaluation of vascular changes with optical coherence tomography angiography after ruthenium-106 brachytherapy of circumscribed choroidal hemangioma
}

 \\ Giovanni Cennamo ${ }^{2}$
}

Received: 5 November 2017 / Revised: 3 March 2018 / Accepted: 19 March 2018 / Published online: 18 May 2018

(c) The Royal College of Ophthalmologists 2018

\begin{abstract}
Purpose To describe the vascular changes of circumscribed choroidal hemangioma (CCH) after ruthenium-106 brachytherapy using optical coherence tomography angiography (angio-OCT).

Methods Seven eyes of 7 patients diagnosed with symptomatic CCH who underwent ruthenium-106 plaque radiotherapy were included in the study. Patients underwent complete ophthalmic examination, bulbar echography and angio-OCT. The same protocol was applied 1 year after ruthenium-106 brachytherapy.

Results At baseline, the mean best-corrected visual acuity (BCVA) in affected eyes was $0.67 \pm 0.14 \log$ AAR, and the mean tumor thickness was $4.46 \pm 0.91 \mathrm{~mm}$ measured by standardized A-scan echography. After ruthenium-106 plaque treatment, the mean BCVA increased to $0.17 \pm 0.10 \operatorname{logMAR}(p=0.001)$ and the mean tumor thickness to $2.29 \pm 0.71 \mathrm{~mm}(p=0.003)$. Moreover, there was a significant reduction of the tumor basal diameter $(p=0.009)$. Intra-tumor angio-OCT measurements revealed a statistically significant reduction of the vessel and flow areas $(p=0.006$ and $p=0.002$, respectively) 1 year after brachytherapy.

Conclusions Angio-OCT is a non-invasive reliable method with which to evaluate the vascular features of $\mathrm{CCH}$ and consequently to monitor changes after conservative treatment.
\end{abstract}

\section{Introduction}

Circumscribed choroidal hemangioma $(\mathrm{CCH})$ is a benign vascular tumor that appears clinically as a round or oval orange-red mass usually posterior to the equator [1]. Ascan echography of $\mathrm{CCH}$ shows high internal reflectivity,

Gilda Cennamo

xgilda@hotmail.com

1 Eye Clinic, Department of Public Health, University of Naples Federico II, Naples, Italy

2 Department of Neurosciences, Reproductive Sciences and Dentistry, University of Naples Federico II, Naples, Italy

3 Department of Advanced Biomedical Sciences, University of Naples Federico II, Naples, Italy

4 Functional and Morphologic Department of Radiotherapy and Legal Medicine, University of Naples "Federico II", Naples, Italy

5 Institute of Biostructure and Bioimaging, National Research Council (CNR), Naples, Italy while B-scan echography shows a dome-shape hyperreflective mass. The tumor is often asymptomatic initially, but will eventually cause a decrease in visual acuity and metamorphopsia associated with fluid exudation [2]. Asymptomatic patients are not treated but kept under observation. Various procedures are used to treat symptomatic serous retinal detachment associated with choroidal hemangiomas, namely, photodynamic therapy, transpupillary thermotherapy, plaque brachytherapy, external beam and proton beam radiation, and stereotactic radiosurgery $[3,4]$. The goal of treatment is resolution of the macular edema. Any decrease in tumor size is a benefit but is not the primary purpose of treatment [5]. Choroidal hemangioma with extensive subretinal fluid, in which neither photodynamic therapy nor transpupillary thermotherapy would be advised, is generally treated with brachytherapy. Brachytherapy with palladium-103, cobalt60 , ruthenium-106, and iodine-125 have all been successfully used to treat $\mathrm{CCH}$ [6-9].

Optical coherence tomography angiography (angio-OCT) is a non-invasive procedure with which to image the retinal 




Fig. 1 Circumscribed choroidal hemangioma before and 1 year after treatment with ruthenium plaque radiotherapy. a Multicolor image of a choroidal circumscribed hemangioma. b EDI-OCT scan through the tumor before treatment shows low-to-medium homogeneous reflective signals from the lesions and elevation of retinal pigment epithelium with intra-subretinal fluid and cystoid macular edema. c EDI-OCT

vasculature without the injection of dye [10]. The aim of this study was to use angio-OCT to evaluate the vascular changes of $\mathrm{CCH}$ after ruthenium-106 brachytherapy [11].

\section{Methods}

Seven eyes of 7 patients, diagnosed with $\mathrm{CCH}$, who underwent ruthenium-106 plaque radiotherapy at the Eye Clinic of the University of Naples "Federico II" from January 2016 to April 2017 were included in this prospective study. The study adhered to the tenets of the Declaration of Helsinki and was approved by the University of Naples "Federico II" Institutional Review Board (IRB)/Ethics Committee (Italy). Patients who were enrolled in the study underwent complete ophthalmic examination, including best-corrected visual acuity (BCVA), according to the scan through the tumor after treatment shows resolution of intrasubretinal fluid and of cystoid macular edema. d Angio-OCT revealed a network of variably sized interconnected vessels in the hemangioma, which appeared larger than the surrounding normal choroidal vessels. e Angio-OCT image showing reduced vessel area after treatment

standard Early Treatment of Diabetic Retinopathy Study (ETDRS) recorded and converted to $\log$ MAR values, fundus examination, bulbar echography and angio-OCT. The same protocol was applied 1 year after 106 ruthenium plaque treatment. Exclusion criteria were clinically relevant opacities of the optic media and low quality angio-OCT images, preexisting macular diseases, pathologic myopia, history of ocular surgery, previous diagnosis of glaucoma. Ancillary test findings were recorded at the initial visit, namely, tumor vessel area and flow area, diameter, thickness, presence or absence of intraretinal and subretinal fluid, and central macular thickness. Patients were examined 1, 3, 6 and 12 months and yearly thereafter. A- and B-scan ultrasonography, and angio-OCT were repeated at each follow-up visit. 


\section{Optical coherence tomography angiography}

Angio-OCT scans were performed using the commercially available RTVue XR Avanti with AngioVue system (Optovue, Fremont, CA, USA), which is based on splitspectrum amplitude decorrelation angiography. Segmentation was manually adjusted to obtain clear images. The tumor vessel area was defined as the percentage area occupied by the large vessels and by the microvasculature in the region analyzed. Angio-OCT revealed a network of variably sized interconnected vessels in the hemangioma, which appeared larger than the surrounding normal choroidal vessels.

The flow area was measured by summing the pixel area to the active vascular flow in the cleaned region selected by the operator on the retinal angiogram. For each eye analyzed, the software automatically calculates vessel area in the whole scanned area and in all sections of the grid. In the case of eyes with a $\mathrm{CCH}$ located at the posterior pole, an $8 \times 8 \mathrm{~mm}$ scan was also performed over the lesion, vessel and flow areas of the tumor using a specific measurement tool of the OCT software. Two expert examiners (GL.C, C. $\mathrm{R}$ ) independently assessed the manual measurements of these parameters; a third investigator (G.C) reassessed measurements that varied by more than $10 \%$ between each independent measurement of the tumor area. Poor-quality images with a signal strength index less than 50 or image sets with residual motion artifacts (discontinuous vessel pattern or discontinuous disc boundary) were excluded from the analysis.

\section{Bulbar echography}

Tumor mean thickness and basal dimension were measured with standardized bulbar echography before and after treatment. A-scan and B-scan ultrasound were performed with an AVISO-S Echograph (Quantel Medical, ClermontFerrand, France) and 10 and $20 \mathrm{MHz}$ probes.

\section{Statistical analysis}

Statistical analysis was performed with the Statistical Package for Social Sciences (Version 20.0 for Windows; SPSS Inc, Chicago, Ill, USA). The Mann-Whitney $U$ test was used to evaluate differences in tumor flow area and vessel density before and after treatment. The same test was used to evaluate differences in BCVA, tumor basal diameter and tumor thickness before and after ruthenium plaque treatment. A $p$ value of $<0.05$ was considered statistically significant.

\section{Results}

Seven eyes of 7 patients (two women and 5 men) were studied. The mean age of the patients was 49 years (range: 44-54 years). The tumors were located in the posterior pole or in the lower quadrant (Fig., 1a). At baseline, the mean BCVA in affected eyes was $0.67 \pm$ $0.14 \log$ MAR. At standardized A-scan echography, the mean tumor thickness was $4.46 \pm 0.91 \mathrm{~mm}$. Most tumors were located in the left eye ( 5 cases versus 2 cases). The characteristics of patients, tumor features and radiation parameters are summarized in Table 1. 1 year after ruthenium-106 plaque treatment, the mean BCVA increased from $0.67 \pm 0.14$ to $0.17 \pm 0.10 \operatorname{logMAR}(p=$ 0.001 ), and mean tumor thickness decreased from $4.46 \pm$ 0.91 to $2.29 \pm 0.71 \mathrm{~mm}(p=0.003)$. Moreover, the tumor basal diameter decrease from $9.27 \pm 1.35$ to $4.76 \pm 2.0$. The intra-tumor angio-OCT measurements revealed a statistically significant reduction of the vessel and flow areas between baseline and 1 year follow-up $(p=0.006$; $p=0.002$ ) (Table 2). Angio-OCT findings showed that subretinal fluid and cystoid macular edema had resolved in all patients (Fig. 1b, c).

In all cases, the angio-OCT images showed a characteristic pattern constituted by a dense irregular vascular network in the choroid capillary layers similar to a multilobular pattern, which may represent the vessel walls of the tumor. As shown in Fig. 1d, e, posttreatment angio-OCT scans show a reduction of the vascular network.

Table 1 Patients demographics, OCT features and radiation parameters

\begin{tabular}{ll}
\hline Feature & $\begin{array}{l}\text { Number }(\boldsymbol{n} \\
=7)\end{array}$ \\
\hline $\begin{array}{l}\text { Age, mean, } \\
\text { years }\end{array}$ & 49.86 \\
$\begin{array}{l}\text { Female } \\
\text { Right eye }\end{array}$ & 2 \\
$\begin{array}{l}\text { Tumor position } \\
\text { Posterior pole }\end{array}$ & 2 \\
$\begin{array}{l}\text { Lower } \\
\text { quadrant }\end{array}$ & 3 \\
Radiation dose, mean, cGy & 4 \\
$\begin{array}{l}\text { Tumor apex } \\
\text { Tumor base } \\
\text { OCT features, n. } \\
\text { Macular } \\
\text { subretinal fluid } \\
\text { Subfoveal fluid }\end{array}$ & 100 \\
\hline
\end{tabular}


Table 2 Differences in OCT angiography vessel density and echographic featuresin angioma eyes at baseline and at 1 year follow-up

\begin{tabular}{|c|c|c|c|}
\hline & $\begin{array}{l}\text { Patients at baseline } \\
(\text { mean } \pm \mathrm{SD})\end{array}$ & $\begin{array}{l}\text { Patients at } 1 \text { year follow-up } \\
(\text { mean } \pm S D)\end{array}$ & $\begin{array}{l}\text { Baseline vs follow-up ( } p \\
\text { value) }\end{array}$ \\
\hline Tumor vessel area, $\mathrm{mm}^{2}$ & $17.69 \pm 1.49$ & $13.92 \pm 3.32$ & 0.006 \\
\hline Tumor flow area, $\mathrm{mm}^{2}$ & $9.91 \pm 2.30$ & $7.41 \pm 0.61$ & 0.002 \\
\hline Tumor basal diameter, $\mathrm{mm}$ & $9.27 \pm 1.35$ & $4.76 \pm 2.02$ & 0.009 \\
\hline Tumor thickness, mm & $4.46 \pm 0.91$ & $2.29 \pm 0.71$ & 0.003 \\
\hline BCVA, LogMar & $0.67 \pm 0.14$ & $0.17 \pm 0.10$ & 0.001 \\
\hline
\end{tabular}

\section{Discussion}

To our knowledge, this is the first study to use angio-OCT to evaluate vascular changes after ruthenium-106 brachytherapy of $\mathrm{CCH}$. Classically, spectral-domain optical coherence tomography (SD-OCT) is the procedure used to visualize secondary changes in the retina and retinal pigment epithelium (RPE) in $\mathrm{CCH}$, and can identify macular edema and subretinal fluid $[11,12]$. Heimann et al. described the $\mathrm{CCH}$ features visualized with SD-OCT as a homogenous hyporeflective appearance with intrinsic spaces in the inner surface of the tumor in which the $\mathrm{CCH}$ can be distinguished from the normal surrounding choroid [13]. Enhanced depth imaging (EDI)-OCT showed CCH as a lesion with a smooth, sloping anterior surface without choriocapillaris compression [12, 14]. Other authors described $\mathrm{CCH}$ in EDI-OCT images as a medium-to-low reflective band with a homogeneous signal and intrinsic spaces that possibly correspond to vascular spaces $[15,16]$. As we recently reported, angio-OCT imaging showed a dense irregular vascular network in the choroid capillary layers of CCHs [17].

Circumscribed choroidal hemangioma can lead to severe vision loss in about $50 \%$ of cases secondary to long-term macular edema. The earlier treatment is implemented, the better the visual outcome. Brachytherapy is usually the treatment of choice for $\mathrm{CCH}$ [6-9]. Ruthenium-106 has a lower penetration rate than other radioisotopes and thus has the advantage of delivering a limited range of electrons to a very small area. This treatment results in a decrease of subretinal fluid caused by shrinkage of the tumor. The exudation of subretinal fluid from the tumor may be linked to the blood flow pattern within it [17]. Consistent with previous studies $[9,18]$, the subretinal fluid and cystoid macular edema resolved in all our patients after ruthenium106 plaque treatment. Angio-OCT scans show the vascular cavernous nature of choroidal hemangiomas that clearly differentiates them from the surrounding choroidal vessels. The vaso-occlusive mechanism of brachytherapy, which causes thrombosis of the angiomatous channels, has been demonstrated by angio-OCT $[19,20]$.
This study has several limitations, namely, the relatively low number of patients enrolled, the difficulty in obtaining reliable images from patients, distorted images in eyes with severe macular pathology and image artifacts. The limitations of the technique itself, namely, the inability to reach lesions located beyond the vascular arcades.

In conclusion, angio-OCT is a rapid, non-invasive, highresolution procedure with which to monitor the $\mathrm{CCH}$ vascular features during follow-up after brachytherapy.

\section{Summary}

\section{What was known before}

- Angio-OCT imaging showed a dense irregular vascular network in the choroid capillary layers of $\mathrm{CCH}$.

\section{What this study adds}

- Angio-OCT is a rapid, non-invasive, high-resolution procedure with which to monitor the $\mathrm{CCH}$ vascular features during follow-up after brachytherapy.

\section{Compliance with ethical standards}

Conflict of interest The authors declare that they have no conflict of interest.

\section{References}

1. Madreperla SA, Hungerford JL, Plowman PN, Laganowski HC, Gregory PT. Choroidal hemangioma visual and anatomic results of treatment by photocoagulation or radiation therapy. Ophthalmology. 1997;104:1773-9.

2. Shields CL, Honavar SG, Shields JA, Cater J, Demirci H. Circumscribed choroidal hemangioma: clinical manifestations and factors predictive of visual outcome in 200 consecutive cases. Ophthalmology. 2001;108:2237-48.

3. Mashayekhi A, Shields CL. Circumscribed choroidal hemangioma. Curr Opin Ophthalmol. 2003;14:142-9.

4. Cennamo G, Breve MA, Rossi C, Romano MR, de Crecchio G, Cennamo G. Tranpupillary thermotherapy as a primary treatment 
for circumscribed choroidal haemangioma. Acta Ophthalmol. 2016;94:e167-169.

5. Kivelä T, Tenhunen M, Joensuu T, Tommila P, Joensuu H, Kouri M. Stereostatic radiotherapy of symptomatic circumscribed choroidal hemangiomas. Ophthalmology. 2003;110:1977-82.

6. Singh AD, Kaiser PK, Sears JE. Choroidal hemangioma. Ophthalmol Clin North Am. 2005;18:151-61.

7. López-Caballero C1, Saornil MA, De Frutos J, Bianciotto C, Muiños Y, Almaraz A, López-Lara F, Contreras I. High-dose iodine-125 episcleral brachytherapy for circumscribed choroidal hemangioma. Br J Ophthalmol. 2010;94:470-3.

8. Joshi S, Reddy VAR, Ganesa P, Ali MJ, Naik MN, Honavar SJ. Ruthenium 106 plaque brachytherapy: indications and outcome in ocular tumors. J Cancer Res Ther. 2009;5:S88-9.

9. Naseripour M, Maleki A, Astaraki A, Sedaghat A, Jaberi R, Lee S, Azma Z, Silpa-Archa S. Ruthenium-106 brachytherapy in the treatment of circumscribed choroidal hemangioma. Retina. 2018;38:1024-1030.

10. Spaide RF, Fujimoto JG, Waheed NK. Optical coherence tomography angiography. Retina. 2015;35:2161-2.

11. Shields CL, Pellegrini M, Ferenczy SR, Shields JA. Enhanced depth imaging optical coherence tomography of intraocular tumors: from placid to seasick to rock and rolling topographythe 2013 Francesco Orzalesi Lecture. Retina. 2014;34:1495-512.

12. Heimann H, Jmor F, Damato B. Imaging of retinal and choroidal vascular tumours. Eye (Lond). 2013;27:208-16.

13. Rojanaporn D, Kaliki S, Ferenczy SR, Shields CL. Enhanced depth imaging optical coherence tomography of circumscribed choroidal hemangioma in 10 consecutive cases. Middle East Afr J Ophthalmol. 2015;22:192-7.

14. Torres VL, Brugnoni N, Kaiser PK, Singh AD. Optical coherence tomography enhanced depth imaging of choroidal tumors. Am J Ophthalmol. 2011;151:586-93.

15. Spaide RF, Koizumi H, Pozzoni MC. Enhanced depth imaging spectral-domain optical coherence tomography. Am J Ophthalmol. 2008;146:496-500.

16. Cennamo G, Romano MR, Breve MA, Velotti N, Reibaldi M, de Crecchio G, Cennamo G. Evaluation of choroidal tumors with optical coherence tomography: enhanced depth imaging and OCTangiography features. Eye (Lond). 2017;31:906-15.

17. Takkar B, Azad S, Shakrawal J, Gaur N, Venkatesh P. Blood flow pattern in a choroidal hemangioma imaged on swept-sourceoptical coherence tomography angiography. Indian J Ophthalmol. 2017:65:1240-2.

18. Kubicka-Trząska A, Karska-Basta I, Oleksy P, RomanowskaDixon B. Management of diffuse choroidal hemangioma in Sturge-Weber syndrome with ruthenium-106 plaque radiotherapy. Graefes Arch Clin Exp Ophthalmol. 2015;253:2015-9.

19. Arevalo JF, Shields CL, Shields JA, et al. Circumscribed choroidal hemangioma: characteristic features with indocyanine green videoangiography. Ophthalmology. 2000;107:344-50.

20. Witschel H, Font RL. Hemangioma of the choroid. A clinicopathologic study of 71 cases and a review of the literature. Surv Ophthalmol. 1976;20:415-31. 